\title{
Comment on "Centroid theory of transverse electron-proton two-stream instability in a long proton bunch"
}

\author{
D.V. Pestrikov* \\ Budker Institute of Nuclear Physics, 630090 Novosibirsk, Russian Federation
}

(Received 5 February 2004; published 23 November 2004)

\begin{abstract}
We show that, due to inaccurate calculations with a Volterra integral equation, the results obtained in the commented paper are not true. Such an awkwardness could be avoided if the authors would retain in their calculations initial conditions for the proton bunch centroid.
\end{abstract}

DOI: $10.1103 /$ PhysRevSTAB.7.119201

The authors of the paper in Ref. [1] have studied the beam breakup instability of transverse coherent oscillations of the proton bunch propagating through a stationary electron background. Since such instabilities are presently suspected to pose severe limitations on the operations of high-intensity proton and positron storage rings, the subject of the commented paper is very sound. In addition, an attempt to solve such a problem analytically and for nonuniform linear density of the proton bunch looks very attractive. However, careful reading of the paper results in regrettable confusions. Namely, the solutions to linear differential equations obtained in the commented paper do not depend on initial conditions for the bunch centroid. That is a very unusual result especially for the theory of the beam breakup instabilities.

According to the text of the paper [1], the Fourier amplitudes of the proton bunch centroid $\tilde{Y}\left(z^{\prime}, \omega\right)=$ $\hat{Y}\left(z^{\prime}, \omega\right) G\left(z^{\prime}\right) \exp \left[\left(\Delta_{p}-\Delta_{e}-\omega\right) z^{\prime} / v\right]$ are calculated for the studied problem solving the following homogeneous integral equation [Eq. (36) of Ref. [1]]

$$
\hat{Y}\left(z^{\prime}, \omega\right)=\frac{1}{v^{2} W\left(\omega_{\beta}^{2}-\omega^{2}\right)} \int_{0}^{z^{\prime}} K\left(z^{\prime}, x\right) \hat{Y}(x, \omega) d x .
$$

Here, we use the notations of the paper [1] and only have simplified their Eq. (36) defining the kernel:

$$
K\left(z^{\prime}, x\right)=\Omega^{2}(x) G(x)\left[\Phi\left(z^{\prime}\right) \Psi(x)-\Phi(x) \Psi\left(z^{\prime}\right)\right] .
$$

Now, our Eq. (1) as well as Eqs. (36), (38), and (41) of [1] are the so-called homogeneous Volterra integral equations. Concerning such integral equations it is known (see, e.g., in Ref. [2]) that the homogeneous Volterra integral equations have no solutions except the trivial [i.e., $\hat{Y}\left(z^{\prime}, \omega\right)=0$ ]. This is an exact result. It describes the fundamental differences between solutions to Volterra and Fredholm integral equations and, correspondingly, the fundamental differences between the instabilities of coherent oscillations described by the Volterra or Fredholm integral equations.

*Electronic address: pestrikov@inp.nsk.su
PACS numbers: 29.27.Bd, 41.75.-i, 52.40.Mj, 52.35.Qz

Namely, we remind the reader that the Fredholm integral equations describe the instabilities with the closed feedback coupling between coherent oscillations of the head-on and the tail-on part of the bunch. If particles in the bunch interact via their wakefields, it may occur due to, e.g., synchrotron oscillations of particles. Because of the mentioned closed feedback coupling, such problems have well-defined eigenvalue properties described by relevant dispersion equation with more or less simple solutions. For such problems, positions of the roots of the dispersion equation define the stability conditions of coherent oscillations of the bunch. Unstable modes of the bunch exponentially increase in time with the growth rates which do not depend on both the time and initial conditions for coherent oscillations.

It is not the case of the beam breakup instability which is studied in the commented paper. The beam breakup instabilities occur in the bunches with the suppressed longitudinal mobility of particles. For instance, it can be an instability which develops substantially faster than the periods of the synchrotron oscillations of particles. As far as the wakefields perturb only the oscillations of the tail-on particles while the particles do not exchange their positions in the bunch, the perturbations of the oscillations in the tail regions of the bunch do not affect the oscillations of its head-on particles. That transports the perturbation of coherent oscillations along the bunch. In this case, the oscillations of a bunch sample at a position, e.g., $z$ in the bunch become unstable due to their resonant excitations by the oscillations of previous samples. In particular, the cumulative result of such excitations depends on the distribution of initial conditions for coherent oscillations along the bunch. Mathematically, such instabilities are described by the Volterra integral equations which have no eigenvalue solutions (see, e.g., in Ref. [3]).

This feature of solutions to Eq. (1) can be seen directly from Eq. (41),

$$
\zeta\left(z^{\prime}, \omega\right) \approx \int_{0}^{z^{\prime}} \frac{\Omega^{2}(x) G(x) R^{2}(x)}{v^{2} W\left(\omega_{\beta}^{2}-\omega^{2}\right)} \zeta(x, \omega) d x,
$$

and Eq. (42), 


$$
\begin{gathered}
\zeta\left(z^{\prime}, \omega\right) \approx \exp \left(\frac{i \omega_{\beta}^{2} J\left(z^{\prime}\right)}{\omega^{2}-\omega_{\beta}^{2}}\right), \\
J\left(z^{\prime}\right)=\frac{-i}{\omega_{\beta}^{2} v^{2} W} \int_{0}^{z^{\prime}} \Omega^{2}(x) G(x) R^{2}(x) d x
\end{gathered}
$$

of [1]. Indeed, according to Eq. (3) and for nonsingular solutions, we find $\zeta\left(z^{\prime}=0, \omega\right)=0$. On the other hand, according to Eq. (4) [i.e., Eq. (42) of [1]], we obtain $\zeta(0, \omega) \approx \exp \left(i \omega_{\beta}^{2} J(0) /\left(\omega^{2}-\omega_{\beta}^{2}\right)\right)=1$. This contradiction is avoided solving the homogeneous Eq. (3) correctly. Namely, we should rewrite Eq. (42) of [1] in the following form:

$$
\zeta\left(z^{\prime}, \omega\right) \approx C(\omega) \exp \left(\frac{i \omega_{\beta}^{2} J\left(z^{\prime}\right)}{\omega^{2}-\omega_{\beta}^{2}}\right),
$$

where $C(\omega)$ is the integration constant. Then, the boundary condition $\zeta\left(z^{\prime}=0, \omega\right)=0$ holds if we take $C(\omega)=$ 0 . A similar result is obtained if we replace Eq. (3) by a nonhomogeneous Volterra equation

$$
\zeta\left(z^{\prime}, \omega\right)=g\left(z^{\prime}\right)+\int_{0}^{z^{\prime}} \frac{\Omega^{2}(x) G(x) R^{2}(x)}{v^{2} W\left(\omega_{\beta}^{2}-\omega^{2}\right)} \zeta(x, \omega) d x,
$$

solve this equation using, e.g., iterations, and then calculate the limit of the obtained solution taking $g(x) \rightarrow 0$.

For these reasons, we conclude that solutions to Eqs. (36), (38), and (41) obtained in [1], as well as the results using these solutions, are wrong. Correct expressions describing the beam breakup instability of the bunch centroid can be obtained solving Eq. (32) of [1] with initial conditions. In this case, the Fourier amplitudes of the bunch centroid $\tilde{Y}\left(z^{\prime}, \omega\right)$ are expressed in terms of its initial values $\tilde{Y}\left(z^{\prime}, t=0\right)$ and $\left(d \tilde{Y}\left(z^{\prime}, t\right) / d t\right)_{t=0}$. As a result, relevant Fourier integrals defining the function $Y_{p}\left(z^{\prime}, t\right)$ will differ from that in Eq. (45) of [1]. Although the reader can do all such calculations more or less easily, it is not clear beforehand how strongly the correct solutions will differ from those presented in [1].

[1] Tai-Sen F. Wang, Paul J. Channell, Robert J. Macek, and Ronald C. Davidson, Phys. Rev. ST Accel. Beams 6, 014204 (2003).

[2] F. G. Tricomi, Integral Equations (Interscience, New York, 1957) also available from Dover Publications, New York, 1994, ISBN: .

[3] N.S. Dikansky and D.V. Pestrikov, Physics of Intense Beams and Storage Rings (AIP, New York, 1994). 